\title{
The trajectory of neurotoxic side effects' impact on daily life: a qualitative study
}

\author{
Jenny Drott, Hans Starkhammar, Karin Kjellgren and Carina Berterö
}

\section{Linköping University Post Print}

\section{Tweet}

N.B.: When citing this work, cite the original article.

The original publication is available at www.springerlink.com:

Jenny Drott, Hans Starkhammar, Karin Kjellgren and Carina Berterö, The trajectory of neurotoxic side effects' impact on daily life: a qualitative study, 2016, Supportive Care in Cancer.

http://dx.doi.org/10.1007/s00520-016-3179-1

Copyright: Springer Verlag (Germany)

http://www.springerlink.com/?MUD=MP

Postprint available at: Linköping University Electronic Press

http://urn.kb.se/resolve?urn=urn:nbn:se:liu:diva-126626 
TITLE: THE TRAJECTORY OF NEUROTOXIC SIDE EFFECTS' IMPACT ON DAILY LIFE: A QUALITATIVE STUDY

\begin{abstract}
Authors:
Jenny. Drott. RN, MScN, PhD-student; Hans. Starkhammar, MD, PhD, Associate Professor;

Karin. Kjellgren, RN, MScN, PhD, Professor; Carina. Berterö, RNT, MScN, PhD, Professor
\end{abstract}

Author Affiliations:

Division of Nursing and Department of Medical and Health Sciences, Linköping University, Sweden (Drott, Kjellgren and Berterö); and Regional Cancer Centre, South-East Sweden, Linköping University, Linköping, Sweden (Starkhammar)

\title{
Corresponding author:
}

Jenny Drott. RN, MScN, PhD-student, Division of Nursing and Department of Medical and Health Sciences, Linköping University 58185 Linköping, Sweden (Jenny.Drott@liu.se).

\section{Acknowledgement}

We thank all patients for their participation in this study. We also thank the Swedish Cancer Society, the Department of Oncology and Surgery, Linköping University Hospital, and the Division of Nursing Science at the Department of Medical and Health Sciences for financial support. Further thanks go to all the health professionals in the oncology clinics who were involved in the study in different ways.

\section{Conflict of interest}

The authors have no funding or conflicts of interest to disclose. 


\begin{abstract}
Purpose To explore the experiences of oxaliplatin-induced neurotoxic side effects among patients with colorectal cancer (CRC), and how these side effects influenced their daily lives over time.

Methods To assess neurotoxic side effects, ten patients were repeatedly interviewed. The patients were recruited from two hospitals in south of Sweden, had stage II-III CRC, and had been treated with adjuvant oxaliplatin postoperatively, from November 2013 to October 2015. They had received FOLFOX and XELOX, with a mean total dose of $791 \mathrm{mg}$ oxaliplatin. After completed chemotherapy, at 3,6 and 12 months into the post-treatment phase, 25 interviews were conducted and thematic analysis was used according to Braun and Clarke.

Results Oxaliplatin-induced neurotoxicity affects patients in several ways in the long term. Four themes were identified: Expectation of cure, Dubiety, Normalization and Learn to live with neurotoxity. The findings of this study describe the trajectory of neurotoxicity and its impact on these patients' life situation. The findings confirmed that neurotoxicity is multi-faceted and that the experience of it changes over time.

Conclusion The desire to survive stimulates adaptations and strategies to manage daily life, and patients learn to live with the neurotoxic side effects. This study provides evidence that these patients need individual attention and support during the trajectory of neurotoxic side effects. Current care provision is inadequate due to lack of knowledge of the ways in which neurotoxicity impacts the patient's daily life. This study provides insights that could be used to develop more person-centered care.
\end{abstract}

Keywords adjuvant chemotherapy, colorectal cancer, neurotoxicity, oxaliplatin, qualitative interviews, thematic analysis 


\section{Introduction}

Colorectal cancer (CRC) is one of the most common cancer forms worldwide. The chance of surviving colorectal cancer depends on the stage of the disease. One third of diagnosed colorectal cancer patients have locally advanced disease with one or more lymph nodes affected, indicating a worse prognosis. Postoperative adjuvant chemotherapy with oxaliplatin can increase overall survival for this patient group [1-5]. Oxaliplatin can cause acute and chronic neurotoxicity and can be an intolerable burden for these patients [6-8]. The severity of neurotoxic side effects depends on the dosage and duration of the oxaliplatin dose rate. Dose reduction and complete discontinuation of therapy is common because of fear of debilitating and prolonged neurotoxicity $[9,10]$.

Chronic neurotoxicity is a serious problem for patients who have had a potentially curative resection. Numerous methods to prevent or eliminate neurotoxicity have been tested without success [11, 12]. Guidelines from ASCO state that there are no existing agents to prevent chemotherapy-induced neurotoxicity. For managing existing neurotoxicity, duloxetine has shown positive effects on pain and has also been found to decrease symptoms of tingling and numbness [13]. Chronic neurotoxicity affects the patient's life situation, daily living and self-care in different ways. Neurotoxicity can influence the patient's quality of life negatively, and can be distressing and affect the patient's ability to carry out activities in daily life [14-16]. Increased knowledge is needed about how oxaliplatin-induced neurotoxicity effects influence CRC patients' daily life and life situation over time. There have not been any qualitative studies to provide an existential view of this phenomenon.

This study is a part of a larger project with different designs to survey neurotoxicity. In the present study patients' experiences were captured through interviews, which were conducted when the patients were experiencing neurotoxicity side effects. To our knowledge this is the first long-term follow-up interview study on adjuvant CRC patients describing their experience with oxaliplatin-induced neurotoxicity.

The purpose was to explore CRC patients' experiences of oxaliplatin-induced neurotoxic side effects, and how these side effects influenced their daily lives over time.

\section{Methods}

\section{Study design}

Data was collected through repeated qualitative interviews after the completion of chemotherapy, at 3,6 and 12 months into the post-treatment phase.

\section{Participants and setting}

The patients were chosen using purposeful and consecutive selection, where neurotoxicity side effects were assessed. Patients in the present study were included from November 2013, and were followed to October 2015. The first ten patients who consented to the larger quantitative ongoing study and assessed neurotoxicity side effects were interviewed and followed. Ten patients was screened and all ten participated in this descriptive and explorative 
study. No patients declined to be interviewed. They were recruited from two hospitals in the south of Sweden. Inclusion criteria were: at least 18 years of age, prescribed with oxaliplatin in an adjuvant setting for stage II-III CRC, no known earlier neurotoxicity, ability to speak and understand the Swedish language, and had answered repeated questionnaires to detect neurotoxicity, sent via a self-reporting mobile phone-based system. Exclusion criteria were: neoadjuvant, palliative treatment, known metastasis and reduced cognitive function. No patients had diabetes, medications that could potentially cause peripheral neurotoxicity and no earlier recognized neurotoxicity. None of the patients had previously received neurotoxic chemotherapy. Ten patients who reported neurotoxic side effects in a questionnaire aimed at measuring neurotoxic side effects and their impact on daily life, were followed for one year after treatment, with repeated interviews during that period. These interviews were conducted after they had completed chemotherapy and were 3,6 and 12 months into the post-treatment phase. Patient demographics are shown in Table 1.

All data were treated confidentially. All patients provided oral and written informed consent in line with the declaration of Helsinki (WMA 2013). Ethical approval was obtained from the Regional Ethical Review Board (record no: 2012/301-31).

\section{Procedure}

Patient's neurotoxicity signs were identified using a self-reported mobile phone-based system Circadian Questions (CQ, 21:st Century Mobile AB, http//www.cqmobil.se). Patients' side effects were reported via the patients' own mobile phones and returned and stored in a secured database. Patient's answers concerning neurotoxic side effects from the Oxaliplatin-Associated Neurotoxicity Questionnaire (OANQ) [17], are presented in real-time as web-based graphs (Figure 1). The first author assessed all included patient's side-effects graphs in CQ, twice a day, since they were included in the study. The patients were contacted by telephone every time when they reported neurotoxicity side effects, and interviews were scheduled as set out in the study design. They were also phoned even if they had not registered any neurotoxic side effects in the questionnaire, to check their answers. No patient declined to be interviewed. Each interview began with an introductory question [18], inviting patients to talk about their neurotoxic side effects and how these affected their daily life. Clarifying and follow up questions were used during the interviews. The interviews were audio digitally recorded and lasted between 8-38 minutes (mean 19 minutes). A total of 25 interviews were conducted and the total interview time was 4 hours and 55 minutes (figure 2).

\section{Data analysis}

The interview data according to the aim were analysed with thematic analysis according to Clarke and Braun [19, 20]. Verbatim transcripts of the interviews were processed and initial thoughts and ideas were noted. The transcribed data was read and re-read several times to gain familiarity. Following on from that, codes were identified for features of the data considered pertinent to the research question and the aim. The whole data set was given equal attention so that full consideration could be given to repeated patterns within the data. Next step involved searching for themes. These larger sections of the data consisted of combinations of different codes that may have been very similar or may have been considered to concern the same aspect of the data. All initial codes relevant to the research 
question were incorporated into a theme. To aid the generation of themes, a separate thematic map was made for each patient on every time-point and later a comprehensive map was produced for all ten patients who were included on each time-point. The coded data were used to ensure they formed a coherent pattern and were considered in relation to the data set as a whole. In order to increase the trustworthiness of the findings, the analysis was subjected to peer-debriefing by the research team.

\section{Results}

The analysis of these interviews resulted in four themes (Figure 3). These themes, presented in chronological order, illustrate the trajectory of side-effects and their impact on the patients' daily lives.

\section{Expectation of a cure}

When the chemotherapy was finished the patient's had their minds focused on one thing- being cured. The treatment was hard and they managed it because they trusted in the health care service and they believed in the cure. They still had neurotoxic side effects but they hoped they would disappear. It would just take some time. They looked forward to being healthy again and living a normal life.

“... I have such confidence in health care and that I am becoming well // But lately I have had some more thoughts. Earlier I didn't hesitate at all, I thought that I will be fine and when the treatment (had its impact)...is over, then we go for it (live as usual)” (patient 2)

The patients had expectations of a cure and were living with hope that this terrible situation would come to an end. It was a comforting thought.

\section{Dubiety}

Three months after finishing the treatment the patients had doubts. There was a sense of feeling unsure about the neurotoxic side effects and the prognosis of these symptoms. The patients had doubts about their ability to manage the side effects and also doubts about their future. Different strategies were used to manage the situation. The patients experienced a strange feeling in their bodies and re-prioritized or avoided activities due to the side effects so they could handle their daily life.

"When I walk it feels really strange in some way, it feels like I have filled my socks with sawdust, it is really unpleasant. That's not good, I can tell you, it is an odd unpleasant feeling” (patient 3)

The patients believed that the neurotoxic side effects would fade away. At this point they realized that this was not true. 
"I believed that I should start walking and exercising again, but I haven't been able to. I have some kind of imbalance and feel insecure. I live by a lake and I don't really dare to go down to the boat, because I am afraid of falling" (patient 1)

The patients also described how side effects changed over time. Instead of fading out, the side effects had increased.

"When I had the treatment, I didn't have any problems at all with my feet but now I have plenty of problems with my feet" (patient 6)

\section{Normalization}

After six months the patients started to adapt to the neurotoxic side effects. They had their own intellectual argument to persuade themselves to live a normal life even though they still had symptoms. They were grateful to be alive and also grateful that some of the side effects were not there anymore.

"The only thing left is numbness in my fingertips. But the numbness in my legs is better, that was really hard and I was very worried about my legs!'” (patient 7)

The patients had carried out many adaptations to manage their life situation, and had also conditioned themselves to live with the neurotoxicity. Despite the neurotoxicity in daily life, they argued that they were normalized in their life situation. The adjustment and re-prioritisation of activities made the patients act normally, as they usually did. Sometimes adjustments were not enough; then they just ignore the symptom.

"You become quite used to having numbness in the feet, so that doesn't affect me so much any longer" (patient 6)

"I feel it all the time, but you don't always worry. I try not to think about it all the time" (patient 5)

\section{Learn to live with neurotoxity}

After one year the patients had learned to live with the neurotoxicity. They had learned to ignore the side effects, accepting the distressing feelings.

"You learn to live with this unpleasant feeling in the hands and feet, I mean that I could have been dead today. The choice is easy, I would even put up with more discomfort" (patient 1)

The patients had thoughts about the future, and also about eventual relapse. If they had a relapse, they wondered if they would go through this kind of treatment again. They had learned to stand the side effects, but if they would be worse; that would be hard to take. 
"I don't want the symptoms to increase, I can stand them as they are today and they are possible to live with, but if they should worsen, then it would be no good" (patient 3)

The patients also pointed out that they did not have the same energy as before the chemotherapy, and neurotoxicity sapped their energy. They avoided activities and felt sad about that.

"It is frustrating and it means that I do not have the same spark as before; well, it has affected my daily life" (patient 9)

\section{Discussion}

This study provides an in-depth perspective of patients with advanced CRC and with adjuvant chemotherapy experiences of oxaliplatin-induced neurotoxicity. Postoperative adjuvant chemotherapy with oxaliplatin can increase overall survival for this patient group [1-4], but oxaliplatin can cause neurotoxicity and may be an intolerable burden for these patients. The findings confirmed that neurotoxicity is multi-faceted and the experience changes over time. The cancer disease itself had an impact on patients' lives and they balanced the neurotoxic side effects against their survival. Expectation of a cure after completing treatment was a natural outcome and the patients looked forward to live as usual. These experiences were in agreement with results in other studies of cancer patients [21, 22]. In the present study the patients expressed doubts three months after they had finished chemotherapy. After six months in the post-treatment phase the patients started to adapt to the neurotoxic side effects. One year after concluding chemotherapy the patients had learned to live with the neurotoxicity. These results showing the trajectory are not in agreement with any earlier studies. This could be due to our long term follow up. The post-treatment survivorship can be a distressed period of symptom burden, fatigue and reduced mental well-being [23, 24]. The finding that neurotoxicity influences the patients' daily life is a similar result to earlier studies [14, 15, 25-29], but those studies had cross-sectional cohorts, other disease stages, and different study designs.

Our findings highlight the importance of being aware of neurotoxicity and its impact on the patient's life situation in the first year post-treatment. These patients were in outpatient hospital care, so it seemed to be important to enhance the follow-up of this patient group. In this study we identified the patient's neurotoxic side effects using OANQ [17, 30], via a self-report mobile phone system; an appropriate way for professionals to follow symptom progression. In the future, when the quantitative data also is available from the self-report mobile phone system further analysis will be made from combined qualitative and quantitative perspective.

It is important, early in treatment, to recognize side effects and be aware of their consequences to provide adequate support for patients. In this research study the interviews might have had a therapeutic effect on the patients and their situation. Participation in this study was voluntary and patients were encouraged to share their experiences of neurotoxicity and its impact on daily life. No patients declined to be interviewed, even though the interviews were repeated. The interviews were in addition to the regular care routine, and seemed to be a useful way to support and confirm the patients. 
This study has some limitations such as sample size, but this size was relevant to the study design. A strength could be that the sample included patients of different gender, age and marital status, with a variety of experiences. To enhance the credibility and trustworthiness, first and last author worked on the analysis. All authors jointly discussed the results and came into agreement. The methodological procedures are presented in a way that makes it possible to follow the audit trail. This study has a limited follow-up time period of one year, but this study is unique since it follows the patients with repeated qualitative interviews. Future research, longer follow up period of CRC patients both with quantitative and qualitative methodology is needed.

In conclusion, this is the first study to systematically explore CRC patients' experiences of neurotoxicity over time to define the neurotoxic trajectory. This study provides evidence that these patients need individual attention and support in the trajectory of remaining side-effects. Current care provision is inadequate due to lack of knowledge of the ways in which neurotoxicity impacts the patient's daily life. This study provides insights that could be used to develop more person-centered care. Innovative care and support based on patient-reported neurotoxicity are needed in order to improve quality of life. 


\section{References}

1. De Gramont A, Buyse M, Abrahantes JC et al (2007) Reintroduction of oxaliplatin is associated with improved survival in advanced colorectal cancer. J Clin Oncol 25:3224-3229

2. O'Connell MJ, Mailliard JA, Kahn MJ et al (1997) Controlled trial of fluouracil and low-dose leucovorin given for 6 months as postoperative adjuvant therapy for colon cancer. J Clin Oncol 15:246-250

3. Kuebler JP, Wieand HS, O'Connell MJ et al (2007) Oxaliplatin combined with weekly bolus flurouracil and leucovorin as surgical adjuvant chemotherapy for stage II and III colon cancer: Results from NSABP C-07. J Clin Oncol 25: 2198-2204

4. Yothers G, O’Connell MJ, Allegra CJ et al (2011) Oxaliplatin as adjuvant therapy for colon cancer: Updated results of NSABP C-07 trial, including survival and subset analyses. J Clin Oncol 29:3768-3774

5. Schmoll HJ, Tabernero J, Maroun J et al (2015) Capecitabine Plus Oxaliplatin Compared With Fluorouracil/ Folinic Acid As Adjuvant Therapy for Stage III Colon Cancer: Final Results of the NO16968 Randomized Controlled Phase III Trial. J Clin Oncol 33:3733-3740

6. Seretny M, Currie GL, Sena ES et al (2014) Incidence, prevalence, and predictors of chemotherapy-induced peripheral neuropathy: A systematic review and meta-analysis. Pain. 155:2461-2470

7. André T, Boni C, Mounedji-Boudiaf L et al (2004) Oxaliplatin,fluorouracil, and leucovorin as adjuvant treatment for colon cancer. N Engl J Med 350:2343-2351

8. Mols F, Beijers T, Lemmens V et al (2013) Chemotherapy-Induced Neuropathy and Its Association With Quality of Life Among 2- to 11-Year Colorectal Cancer Survivors: Results From the Population-Based PROFILES Registry. J Clin Oncol 20:2699-2707

9. Vatandoust S, Joshi R, Pittman KB et al (2014) A descriptive study of persistent oxaliplatin-induced peripheral neuropathy in patients with colorectal cancer. Support Care Cancer 22:513-518

10. Velasco R, Bruna J, Briani C et al (2013) Early predictors of oxaliplatin-induced cumulative neuropathy in colorectal cancer patients. J Neurol Neurosurg Psychiatry 85:392-398

11. Sereno M, Gutiérrez-Gutiérrez G, Gómez-Raposo C et al (2014) Oxaliplatin-induced-neuropathy in digestive tumours. Crit Rev Oncol Hematol 89:166-78

12. Hershman DL, Lacchetti C, Dworkin RH et al (2014) Prevention and management of chemotherapy-induced peripheral neuropathy in survivors of adult cancers: American Society of Clinical Oncology clinical practice guideline. J Clin Oncol 32:1941-1967

13. Smith EM, Pang H, Cirrincione $C$ et al (2013) Effect of duloxetine on pain, function, and quality of Support Care Cancer life among patients with chemotherapy-induced painful peripheral neuropathy: a randomized clinical trial. JAMA 309:1359-1367

14. Tofthagen C, McAllister RD, McMillan SC (2011) Peripheral neuropathy in patients with colorectal cancer receiving oxaliplatin. Clin J Oncol Nurs 15:182-188

15. Bakitas M: Background Noise (2007) The Experience of Chemotherapy-Induced Peripheral Neuropathy. Nurs Res 56:323-331

16. Qian H, Yuan C (2012) Factors associated with self-care self-efficacy among gastric and colorectal cancer patients. Cancer Nursing 35:22-31 
17. Leonard G.D, Wright M.A, Quinn M.G et al (2005) Survey of Oxaliplatin-Associated Neurotoxicity Using an Interview-Based Questionnaire in Patients with Metastatic Colorectal Cancer. BMC Cancer 5:116-125

18. Patton MQ (2002) Qualitative research and evaluation methods. $3^{\text {rd }}$, London, UK, Sage Publications Ltd

19. Braun V, Clarke V (2013) Successful qualitative research; a practical guide for beginners. London, Sage

20. Braun V, Clarke V (2006) Using thematic analysis in psychology. Qualitative Research in Psychology 3:77101

21. Simpson MF, Whyte F (2006) Patients' experiences of completing treatment for colorectal cancer in a Scottish District General Hospital. Eur J Cancer Care 15:172-82

22. Taylor C, Richardson A, Cowley S (2011) Surviving cancer treatment: an investigation of the experience of fear about, and monitoring for, recurrence in patients following treatment for colorectal cancer. Eur J Oncol Nurs 15:243-249

23. Jones JM, Olson K, Catton P et al (2015) Cancer-related fatigue and associated disability in post-treatment cancer survivor. J Cancer Surviv April 16

24. Mosher CE, Winger JG, Given BA et al (2015) Mental health outcomes during colorectal cancer survivorship: a review of the literature. Psychooncology Aug 27

25. Bennett BK, Park SB, Lin C et al. (2012). Impact of oxaliplatin-induced neuropathy: a patient perspective. Support Care Cancer, 20(11), 2959-2967

26. Briani C, Argyriou AA, Izquierdo C et al (2014). Long-term course of oxaliplatin-induced polyneuropathy: a prospective 2-year follow-up study. J Periph Nerv System 19, 299-306

27. Tofthagen C, Gonzalez L, Visovsky C et al. (2013). Self-management of oxaliplatin-related peripheral neuropathy in colorectal cancer survivors. Chemother Res Pract. 2013, 547932

28. Tofthagen C, Donovan KA, Morgan MA et al (2013). Oxaliplatin-induced peripheral neuropathy's effects on health-related quality of life of colorectal cancer survivors. Supportive Care in Cancer, 21(12), 3307-3313

29. Padman S, Lee J, Kumar R et al (2015). Late effects of oxaliplatin-induced peripheral neuropathy (LEON)cross-sectional cohort study of patients with colorectal cancer surviving at least 2 years. Support Care Cancer, 23, 861-869

30. Gustafsson E, Litström E, Berterö C et al (2015) Reliability testing of oxaliplatin-associated neurotoxicity questionnaire (OANQ), a pilot study. Support Care Cancer 2016; 24(2):747-54 


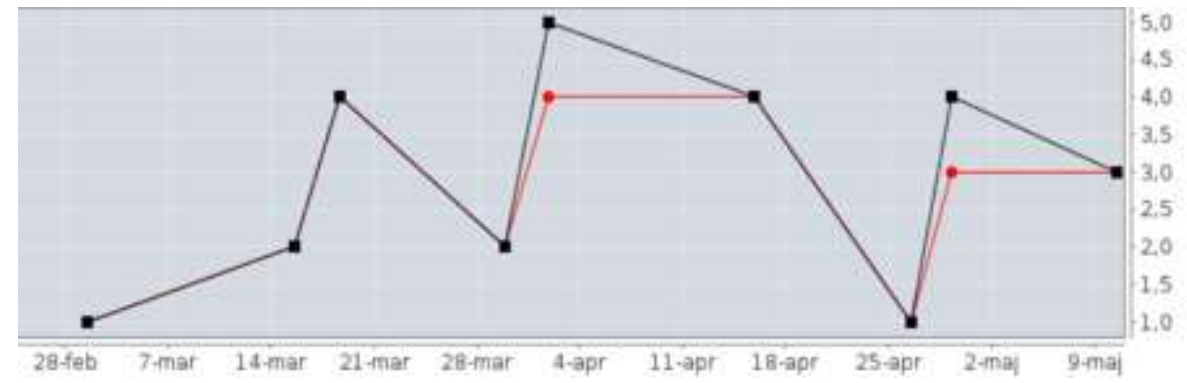

A) Difficulty feeling cold things (Upper extremity)

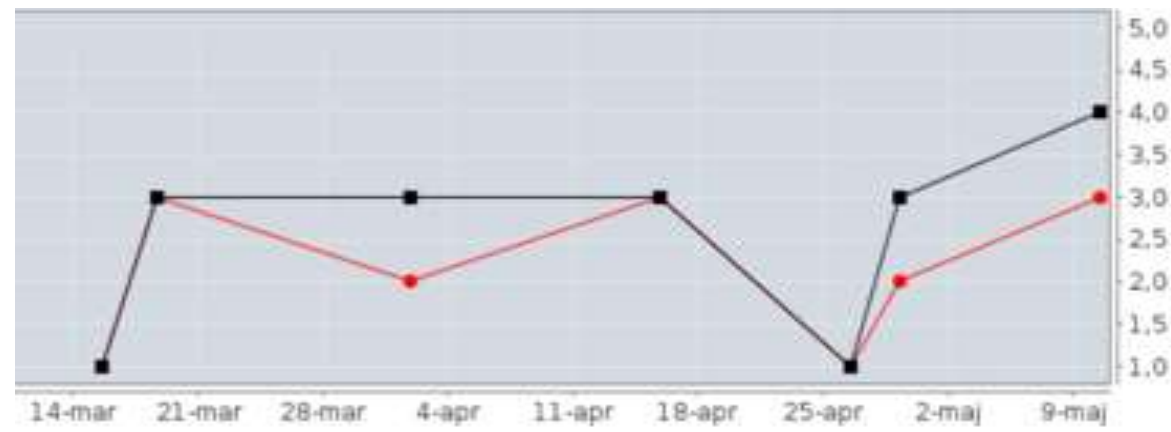

B) Jaw pain (Oral/Facial)

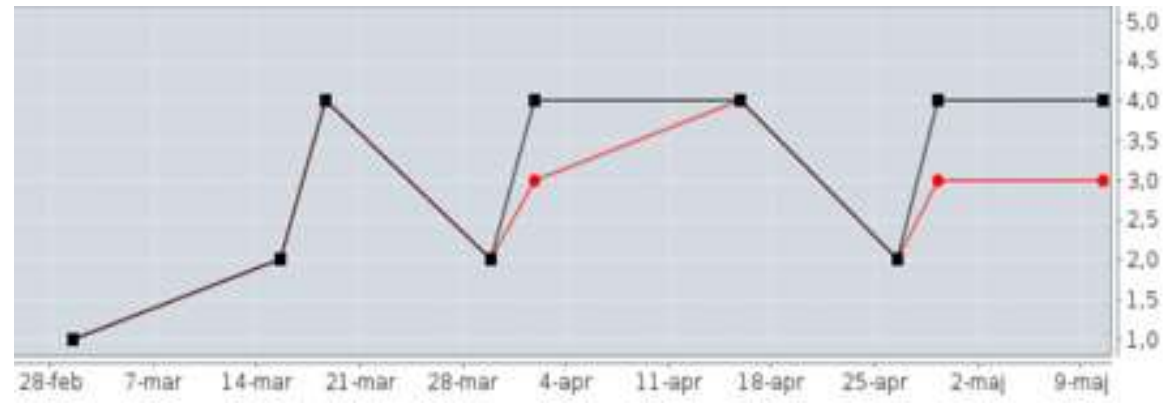

C) Burning pain or discomfort with cold (Upper extremity)

Figure 1. Presenting web-based graphs from one patients illustrating three questions (Oxaliplatin-Associated Neurotoxicity Questionnaire, total 29 items) on self-reported neurotoxic side-effects over 70 days in the mobile phone-based system. Patients' self-reported grade of neurotoxicity from scale 1.0-5.0 (black lines), patients' self-reported grade of neurotoxicity impact of daily life from scale 1.0-5.0 (red lines). Answers are only visualized in the graphs when the patients had neurotoxic side effects over 1.0. 


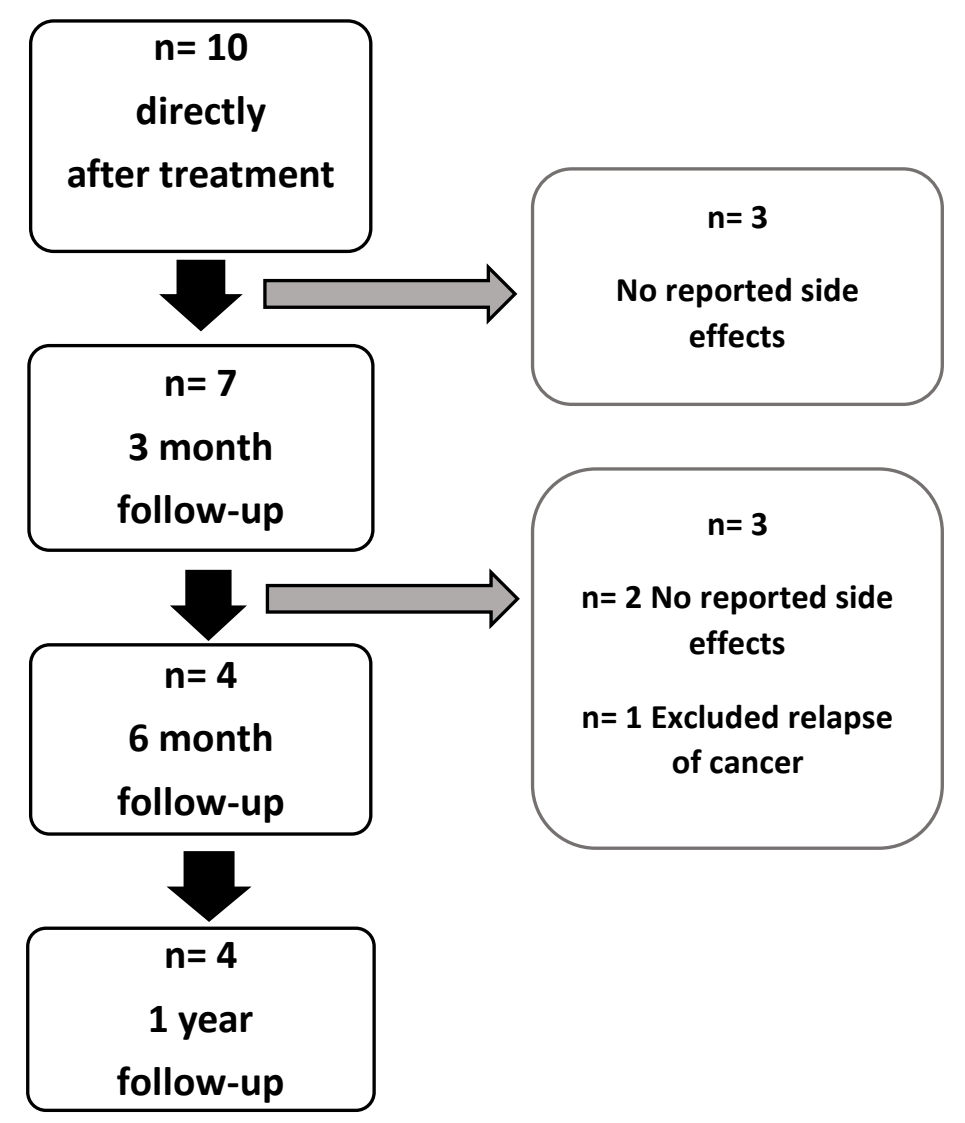

Figure 2. Ten patients with reported neurotoxic side effects were interviewed. Interviews were repeated with patients with remaining side effects: twenty-five interviews in total. 

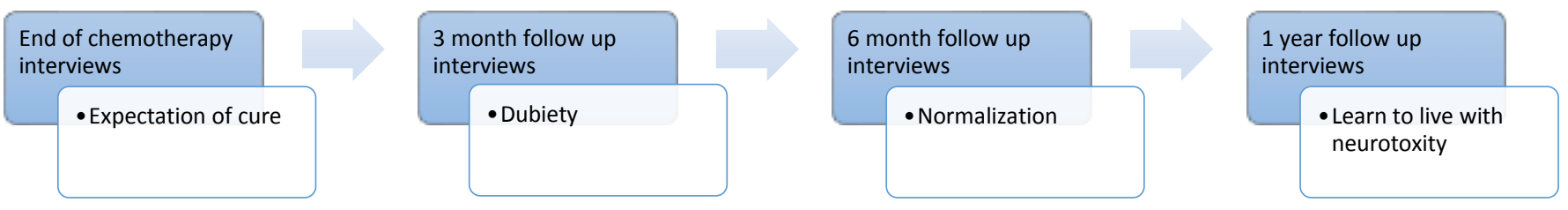

Figure 3. The themes illustrates the trajectory of side effects impact on these patients' daily lives. 
Table 1 Patient demographics

Clinical information

Age (years) range, median/mean

(44-68) 65/61

Gender, $n$

Male 3

Female

7

Social status, $n$ Living alone $\quad 2$

Living with life partner $\quad 8$

Children at home 2

Type of malignancy, $n$

Colon

9

Rectal

1

Education level, $n$

Less than high school 2

High school or college 5

University 3

Chemotherapy, $n$

FOLFOX 1

XELOX 9

Oxaliplatin mg (min/max) mean

$(175 / 1500) 791$

Number of cycles with oxaliplatin

$(1 / 7) 4,6$

$(\min / \max )$ mean 